\title{
Real-Time Driver's Monitoring Mobile Application through Head Pose, Drowsiness and Angry Detection
}

\author{
Hamimah Ujir \\ Faculty of Computer Science and \\ Information Technology, \\ Universiti Malaysia Sarawak \\ Sarawak, Malaysia \\ uhamimah@unimas.my
}

\author{
Ee Min Jee \\ Faculty of Computer Science and \\ Information Technology, \\ Universiti Malaysia Sarawak \\ Sarawak, Malaysia \\ kimijiejie@gmail.com
}

\author{
Mohammad Farhaan Iqbal \\ Faculty of Computer Science and \\ Information Technology, \\ Universiti Malaysia Sarawak \\ Sarawak, Malaysia \\ farhaan.professional@gmail.com
}

\author{
Qan Khai Mun \\ Faculty of Computer Science and Information Technology, \\ Universiti Malaysia Sarawak \\ Sarawak, Malaysia \\ jayqan96@hotmail.com
}

\author{
Irwandi Hipiny \\ Faculty of Computer Science and Information Technology, \\ Universiti Malaysia Sarawak \\ Sarawak, Malaysia \\ mhihipni@unimas.my
}

\begin{abstract}
The current driver's monitoring system requires a set-up that includes the usage of a variety of camera equipment behind the steering wheel. It is highly impractical in a real-world environment as the set-up might cause annoyance or inconvenience to the driver. This project proposes a framework of using mobile devices and cloud services to monitor the driver's head pose, detect angry expression and drowsiness, and alerting them with audio feedback. With the help of a phone camera functionality, the driver's facial expression data can be collected then further analyzed via image processing under the Microsoft Azure platform. A working mobile app is developed, and it can detect the head pose, angry emotion, and drowsy drivers by monitoring their facial expressions. Whenever an angry or drowsy face is detected, pop-up alert messages and audio feedback will be given to the driver. The benefit of this mobile app is it can remind drivers to drive calmly and safely until drivers manage to handle their emotions where anger or drowsy is no longer detected. The performance of the mobile app in classifying anger emotion is achieved at $96.66 \%$ while the performance to detect driver's drowsiness is $82.2 \%$. On average, the head pose detection success rate across the six scenarios presented is $85.67 \%$.
\end{abstract}

Keywords—driver's monitoring, facial expression, head pose, mobile application, real-time

\section{INTRODUCTION}

Numerous studies on developing face processing application on a mobile phone are available and developed for different purposes, i.e., entertainment, security. Among examples of face processing mobile application are face recognition, face tracking, and facial expression recognition. These potential applications can be extended to driver's monitoring application.

The idea of a driver's monitoring system is rather to monitor safety during driving. Three common reasons for road accidents are (i) fatigue which encompasses drowsiness; (ii) aggressive driving style and [iii] driver's distraction. There are several causes of distraction and inattention such as mobile phone usage, eating, and adjusting the radio channel and volume. When it comes to distraction and head tracking technology, there is a strong link between the driver's head metrics to the level of attention during driving. Studies in [1] shows that gaze regions from the tasks of the same activity class would be visually similar. Therefore, observing the driver's gaze region may reflect the attentiveness of the driver. Distracted driving like messaging or talking on the phone are the factors causing road rage. Road rage is related to the increment of the driver's emotional level of anger. Facial features are then used as the input for emotional detection.

Drowsiness on the road may be due to circadian-rhythm disorders and diseases that may affect even a well-rested driver. Over the decades the use of the percentage of eye closure (PERCLOS) metric as an evaluation standard for detection of fatigue drivers within a finite period is persistent. Apart from eye closure detection, yawning is also a sign of fatigue which leads to drowsiness. Another study suggests that frequent yawning followed by burning feeling in the eyes and hard to keep them open is a sign of fatigue [2].

There are two approaches used to measure the biometrics of the vehicle driver, either it is an instrumentation of the car or instrumentation of the driver. Instrumenting the car, meaning adding sensors to the car to understand the driver as in [3][4], which falls short as it is difficult to develop to adapt to each car model as well as difficult to implement into the car if not pre-installed by design in the factory. Meaning that the existing car model is possibly left out of this solution. Instrumenting the driver means adding sensors and apparatus to the driver's body as explored in [5][6]. This category of solution also falls short when it comes to development. The actual practicality and feasibility of always wearing sensors on your body are less attractive. It often requires bespoke hardware, difficult access to every driver, and complex software tuned for each set of hardware.

According to [7], the objective of driving monitoring and assistance systems (DMAS) is to keep an eye on the driver's status and to provide necessary assistance for safe and comfortable driving. DMAS considers the three main elements of the driving process, i.e., driver, vehicle, and driving environment. The focus of this paper is one of the elements in the driving process, which is the driver. With a 